\title{
Role of New Echocardiographic Technologies in Diagnosing the Classic Subtype of Arrhythmogenic Cardiomyopathy
}

\author{
Xihui Liu ${ }^{1}$, Yonghuai Wang ${ }^{2}$, Guangyuan $\mathrm{Li}^{3}$, Lixin $\mathrm{Mu}^{2}$, Shuo $\mathrm{Liu}^{2}$, Chunyan $\mathrm{Ma}^{4}$, and \\ Jun Yang ${ }^{3}$ \\ ${ }^{1}$ China Medical University First Hospital \\ ${ }^{2}$ Affiliation not available \\ ${ }^{3}$ The First Affiliated Hospital of China Medical University \\ ${ }^{4}$ The First Hospital of China Medical University,
}

May 8, 2020

\begin{abstract}
Arrhythmogenic cardiomyopathy (AC) is a rare inherited myocardial disorder, a cause of sudden death in young people and atheletes; biventricular involvement is often involved. We report on a case of AC diagnosed with multimodality imaging, including transthoracic echocardiography (TTE), two-dimensional strain echocardiography (2DSE), real-time three-dimensional echocardiography (RT-3DE) and cardiac magnetic resonance (CMR) imaging. Especially, combination of 2DSE and RT-3DE was utilized in the diagnosis of AC. In a word, we review the cardiac anomalies associated with AC by multiple imaging techniques and highlight the supplementary role of new echocardiography techniques diagnosing AC when CMR contraindications exist for $\mathrm{AC}$ patients.
\end{abstract}

Title: Role of New Echocardiographic Technologies in Diagnosing the Classic Subtype of Arrhythmogenic Cardiomyopathy

Running head: New Echocardiographic Technologies' Role in AC

Authors: Xihui Liu ${ }^{1}$, Yonghuai Wang ${ }^{1}$, Guangyuan $\mathrm{Li}^{1}$, Lixin $\mathrm{Mu}^{1}$, Shuo Liu ${ }^{1}$, Chunyan Ma ${ }^{1, *}$, Jun Yang ${ }^{1}$

${ }^{1}$ Department of Cardiovascular Ultrasound, The First Hospital of China Medical University, Shenyang, Liaoning, China

CORRESPONDING AUTHOR:

Chunyan Ma

E-mail address: cmu1h_mcy@126.com

Address: First Hospital of China Medical University, No.155 Nanjingbei Street, Shenyang, Liaoning 110001, China

Tel/fax:+86-24-83282114

\section{AUTHOR CONTRIBUTIONS:}

Xihui Liu is charged of drafting article,concept and design; Yonghuai Wang is charged of interpretation; Shuo Liu is charged of collecting figures, Guangyuan $\mathrm{Li}$ and Lixin $\mathrm{Mu}$ are charged of managing of figures; Jun Yang is charged of approval of article; Chunyan Ma is charged of critical revision of article. 
ABSTRACT : Arrhythmogenic cardiomyopathy (AC) is a rare inherited myocardial disorder, a cause of sudden death in young people and atheletes; biventricular involvement is often involved. We report on a case of AC diagnosed with multimodality imaging, including transthoracic echocardiography (TTE), twodimensional strain echocardiography (2DSE), real-time three-dimensional echocardiography (RT-3DE) and cardiac magnetic resonance (CMR) imaging. Especially, combination of 2DSE and RT-3DE was utilized in the diagnosis of AC. In a word, we review the cardiac anomalies associated with AC by multiple imaging techniques and highlight the supplementary role of new echocardiography techniques diagnosing $\mathrm{AC}$ when CMR contraindications exist for AC patients.

KEY WORDS : arrhythmogenic right ventricular dysplasia, cardiomyopathy, strain, three-dimensional echocardiography

\section{INTRODUCTION :}

Arrhythmogenic right ventricular dysplasia (ARVD) is an inherited cardiomyopathy, which mainly affects the right ventricle $(\mathrm{RV})^{[1]}$. The left ventricle $(\mathrm{LV})$ involvement was considered merely as a late manifestation. Therefore, the disorder is gradually being mentioned to as arrhythmogenic cardiomyopathy (AC) ${ }^{[2]}$. Recently, 3 patterns of AC had been recognized: the classic subtype, with its acknowledged dominance of $\mathrm{RV}$; the biventricular variant, recognized by similar involvement of both ventricles; and left dominant $\mathrm{AC}^{[3]}$. Diagnoses of ARVD are based on revealing RV abnormalities, which include comprehensive assessment of electrical, anatomical, and functional abnormalities ${ }^{[4]}$. Despite it has been suggested that ARVD with LV involvement might be called $\mathrm{AC}$, there has even no clear guidelines in the diagnosis of classic subtype of AC. Besides, diagnosis of minor LV involved AC, classic subtype, has no explicit criterion yet. We report on a case of a 39-year-old man with classic subtype AC evaluated by multiple imaging technologies, including electrocardiogram (ECG), transthoracic echocardiography (TTE), two-dimensional strain echocardiography (2DSE), real-time three-dimensional echocardiography (RT-3DE) and cardiac magnetic resonance (CMR) imaging. Especially, we here introduce the first application of 2DSE and RT-3DE on the diagnosis of classic AC by contrasting to CMR.

\section{CASE REPORT :}

A 39-year-old male presented with paroxysmal heart palpitations for 7 months and a recurrence in symptoms for 1 week.

Electrocardiogram showed frequent ventricular premature, ventricular tachycardia (VT) (Fig.1A), complete right bundle branch block (RBBB) and inverted $\mathrm{T}$ wave in leads V1-V6 (Fig.1B).

Echocardiography showed no abnormalities in the standard apical four-chamber view and parasternal four chamber view (Fig.2A, B), while bulge and akinesia was observed at the apical segment of the RV lateral wall on right ventricular-focused view (Fig.3A), and basal segment of the RV inferior wall on RV inflow tract view (Fig.3B), with respectively decreased RV longitudinal strain (RVLS, $-7.8 \% ;-16 \%$, Fig.3C, D). Meanwhile, RV fractional area change (FAC, 31\%), tricuspid annular displacement (TAPSE,16mm) and RV ejection fraction on RT-3DE (RT-3DE-RVEF, 34\%) reduced. Bulge at apex of RV was clearly unfolded on RT-3DE (Fig.3E).

Although the LV size and systolic function were within normal range on conventional echocardiography(with LVEF, 60\%), with the decreased LV global longitudinal strain (LV-GLS, -16.4\%), especially at apex, posterior septum and posterior wall of LV (Fig.3F), which was consistent with the region of cardiac magnetic resonance (CMR-LGE) presentation on the 4-chamber long axis view (Fig.4). That CMR-LGE demonstrated apex and lateral wall of RV and posterior septum, posterior lateral wall of LV myocardial replacement of fatty issues. According to the 2010 ARVC diagnostic standard ${ }^{[5]}$ and 2017 experts consensus document of the European Association of Cardiovascular Imaging ${ }^{[6]}$, this patient was diagnosed with classic subtype of AC conforming to four main, one minor criteria and LV involvement. After conducted an implantable cardioverter defibrillator implantation, the patient got relieved at symptoms.

\section{DISCUSSION :}


$\mathrm{AC}$ is a relatively rare hereditary cardiomyopathy involving both ventricles which primary clinical presentations include repeated attacks of exercise-related ventricular tachycardia, syncope and sudden death.

ECG, TTE and CMR imaging have been well-established diagnostic methods. And CMR imaging is seen as relatively "golden criterion". In this case, typical ventricular arrhythmias (frequent ventricular premature, ventricular tachycardia, RBBB and T wave inversion in leads V1-V6) were demonstrated on ECG. Because ventricular tachycardias with a RBBB morphology can be the first presentation of left dominant or classic subtype of $\mathrm{AC}^{[7]}$. Hereby, we speculate that both ventricles of this patient might be involved. TTE was performed and typical signs of RV dilation, regional wall thinness, and several areas of wall motion abnormalities in the RV were observed. However, the LV was neither dilated nor thinned, with an apparently normal diastolic and systolic function (LVEF, 60\%). No evident wall motion abnormality were observed in the LV. Accordingly, conventional echocardiography is often unable to detect minor LV involvement discovered on CMR imaging, because these abnormalities are not regularly related to motion dysfunction ${ }^{[8,9]}$.

However, on 2DSE, both the RV and LV regional longitudinal strain were decreased, which involved areas were consistent with the CMR-LGE involved regions. Teske AJ, et al reported 2DSE allows the objective quantification of regional myocardial function ${ }^{[10]}$, which correlates closely with CMR-LGE imaging at fibrotic segments in patients with nonischemic heart disease. What's more, it can even detect regional abnormalities before the appearance of CMR-LGE in LV ${ }^{[9],[11]}$. Despite the capability of 2DSE to detect LV involvement in a large cohort of patients with ARVD is unknown. 2DSE is a relatively novel technique able to quantify regional myocardial strain which closely correlates to CMR-LGE ${ }^{[11]}$.

Besides, RT-3DE provided more precise assessment of RV ejection fraction (RVEF) and more realistic visual effect of regional wall dilation ${ }^{[12]}$. Kim $\mathrm{J}$, et al discovered that in patients with $\mathrm{LV}$ dysfunction, RV volumes and RVEF assessed by RT-3DE associate well with CMR measurements. RT-3DE may be considered as a more widely practical and versatile alternate of CMR for evaluating RV size and function quantificationally in patients with LV dysfunction ${ }^{[13]}$.

\section{CONCLUSION:}

The findings in this case show that widely available techniques can be used to unmask classic subtype of AC. Overall, for suspected biventricular involved AC patients seem to experience more potentially lethal ventricular arrhythmias than ARVD ${ }^{[14]}$. Therefore, the analysis of LV myocardial motion should be taken seriously. However, it is difficult to discover minor LV involvement of ARVD patients only through TTE. Our case highlights that $\mathrm{AC}$ was diagnosed with multimodality imaging technology, minor LV dysfunction detected by 2DSE and decreased RVEF indicated by RT-3DE, both of which corresponding to the results of enhanced CMR imaging. In addition, RT-3DE provided more realistic vision of locally dilated RV wall. These might implied that 2DSE plus RT-3DE might be expected to alleviate the dependency of enhanced CMR for suspected classic subtype of AC patients, especially when some contraindications of enhanced CMR imaging, for example, patients with metal implants, claustrophobia, a restless child or elderly person, exist. Meanwhile, large cohort researches of patients with AC are expected to confirm our findings.

\section{REFERENCES}

[1] Romero J, Mejia-Lopez E, Manrique C, et al. Arrhythmogenic Right Ventricular Cardiomyopathy (ARVC/D): A Systematic Literature Review[J]. Clin Med Insights Cardiol. 2013;7:97-114.

[2] Corrado D, Basso C, Judge DP. Arrhythmogenic Cardiomyopathy[J]. Circ Res. 2017;121(7):784-802.

[3] E F, R M, L C, et al. Importance of cardiac magnetic resonance findings in the diagnosis of left dominant arrythmogenic cardiomyopathy[J]. Revista espanola de cardiologia (English ed). 2020.

[4] Marcus FI, McKenna WJ, Sherrill D, et al. Diagnosis of arrhythmogenic right ventricular cardiomyopathy/dysplasia: proposed modification of the Task Force Criteria[J]. Eur Heart J. 2010;31(7):806-14.

[5] Marcus FI, McKenna WJ, Sherrill D, et al. Diagnosis of arrhythmogenic right ventricular cardiomyopathy/dysplasia: proposed modification of the task force criteria[J]. Circulation. 2010;121(13):1533-41. 
[6] Haugaa KH, Basso C, Badano LP, et al. Comprehensive multi-modality imaging approach in arrhythmogenic cardiomyopathy-an expert consensus document of the European Association of Cardiovascular Imaging $[\mathrm{J}]$. Eur Heart J Cardiovasc Imaging. 2017;18(3):237-53.

[7] Bennett RG, Haqqani HM, Berruezo A, et al. Arrhythmogenic Cardiomyopathy in 2018-2019: ARVC/ALVC or Both?[J]. Heart Lung Circ. 2019;28(1):164-77.

[8] Sen-Chowdhry S, Syrris P, Ward D, et al. Clinical and genetic characterization of families with arrhythmogenic right ventricular dysplasia/cardiomyopathy provides novel insights into patterns of disease expression[J]. Circulation. 2007;115(13):1710-20.

[9] Teske AJ, Cox MG, Peterse MC, et al. Case report: echocardiographic deformation imaging detects left ventricular involvement in a young boy with arrhythmogenic right ventricular dysplasia/cardiomyopathy[J]. Int J Cardiol. 2009;135(1):e24-6.

[10] Teske AJ, De Boeck BW, Melman PG, et al. Echocardiographic quantification of myocardial function using tissue deformation imaging, a guide to image acquisition and analysis using tissue Doppler and speckle tracking[J]. Cardiovasc Ultrasound. 2007;5:27.

[11] Weidemann F, Niemann M, Herrmann S, et al. A new echocardiographic approach for the detection of non-ischaemic fibrosis in hypertrophic myocardium[J]. Eur Heart J. 2007;28(24):3020-6.

[12] Velasco O, Beckett MQ, James AW, et al. Real-Time Three-Dimensional Echocardiography: Characterization of Cardiac Anatomy and Function-Current Clinical Applications and Literature Review Update[J]. Biores Open Access. 2017;6(1):15-8.

[13] Kim J, Cohen SB, Atalay MK, et al. Quantitative Assessment of Right Ventricular Volumes and Ejection Fraction in Patients with Left Ventricular Systolic Dysfunction by Real Time Three-Dimensional Echocardiography versus Cardiac Magnetic Resonance Imaging[J]. Echocardiography. 2015;32(5):805-12.

[14] Mast TP, Teske AJ, vd Heijden JF, et al. Left Ventricular Involvement in Arrhythmogenic Right Ventricular Dysplasia/Cardiomyopathy Assessed by Echocardiography Predicts Adverse Clinical Outcome[J]. J Am Soc Echocardiogr. 2015;28(9):1103-13.e9.

FIGURE 1 Frequent VP, VT (A); complete RBBB and inverted T wave in leads V1-V6 (B).

RBBB: complete right bundle branch block; VP:ventricular premature; VT:ventricular tachycardia

FIGURE 2 No significant abnormalities in the standard apical four-chamber view (A) and parasternal four-chamber view (B).

FIGURE 3 Bulge and akinesia was observed at the RV apical segment of the lateral wall on right ventricular-focused view and basal segment of the inferior wall on RV inflow tract view (A, B), with respectively decreased RV regional longitudinal strain (green curve in C, light blue and yellow curve in D); RT-3DE vividly showed apical regional bulge and RT-3DE-RVEF decreased (E); Global longitudinal strain of LV decreased, especially at apex, posterior septum and posterior wall of LV (F).

RV: right ventricle; RT-3DE: Real-time three-dimensional echocardiography; RT-3DE-RVEF: RV ejection fraction on Real-time three-dimensional echocardiography; LV: left ventricle

FIGURE 4 CMR-LGE on both ventricles. Especially, on the 4-chamber long axis view showed CMRLGE in the apical and lateral wall of RV, and posterior septum, posterior lateral wall of LV myocardial replacement of fatty issues, corresponding to the areas of abnormal strain on 2DSE.

CMR-LGE: late-gadolinium-enhancement on cardiac magnetic resonance; LV: left ventricle; RV: right ventricle; 2DSE: two-dimensional strain echocardiography 

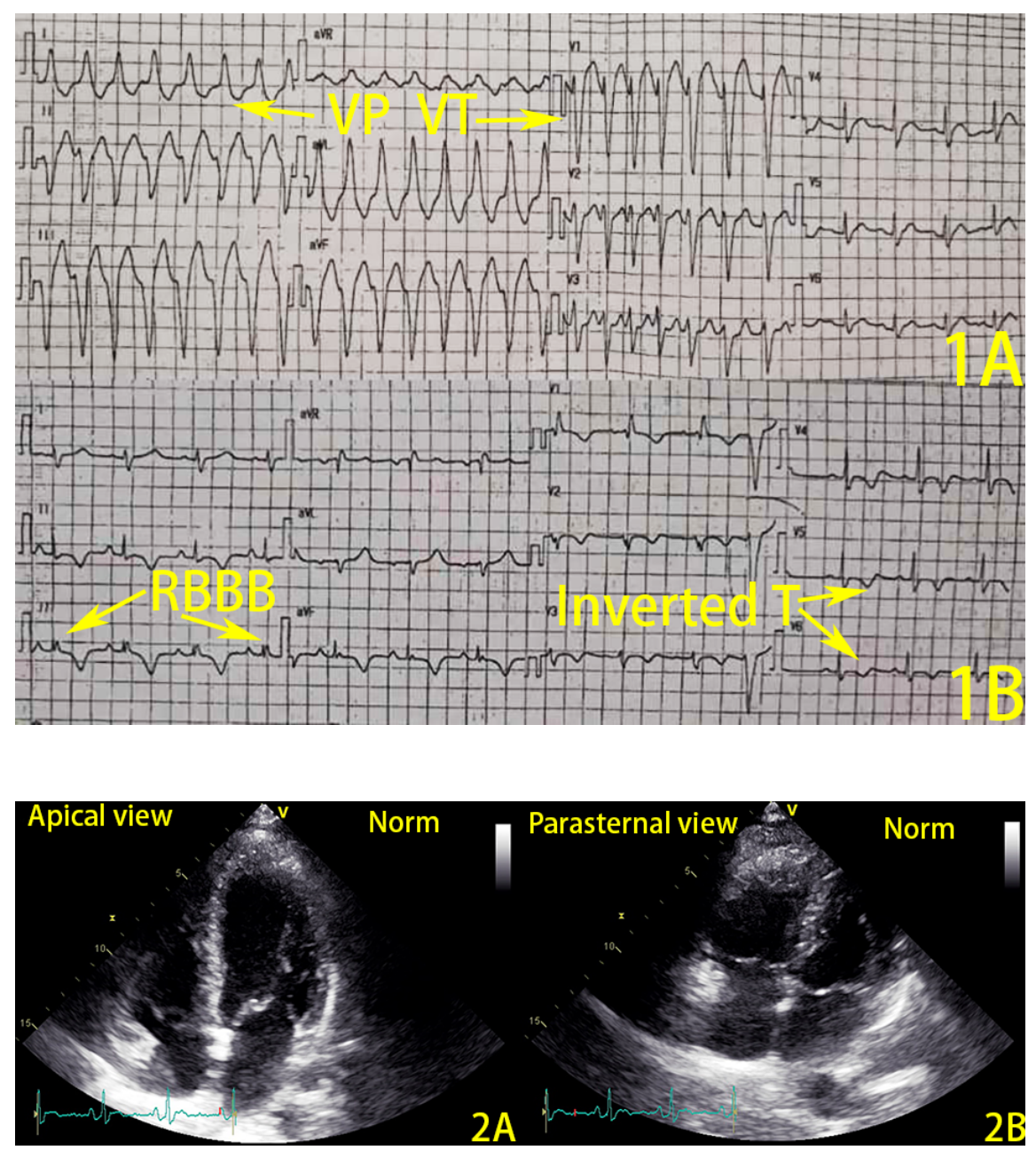


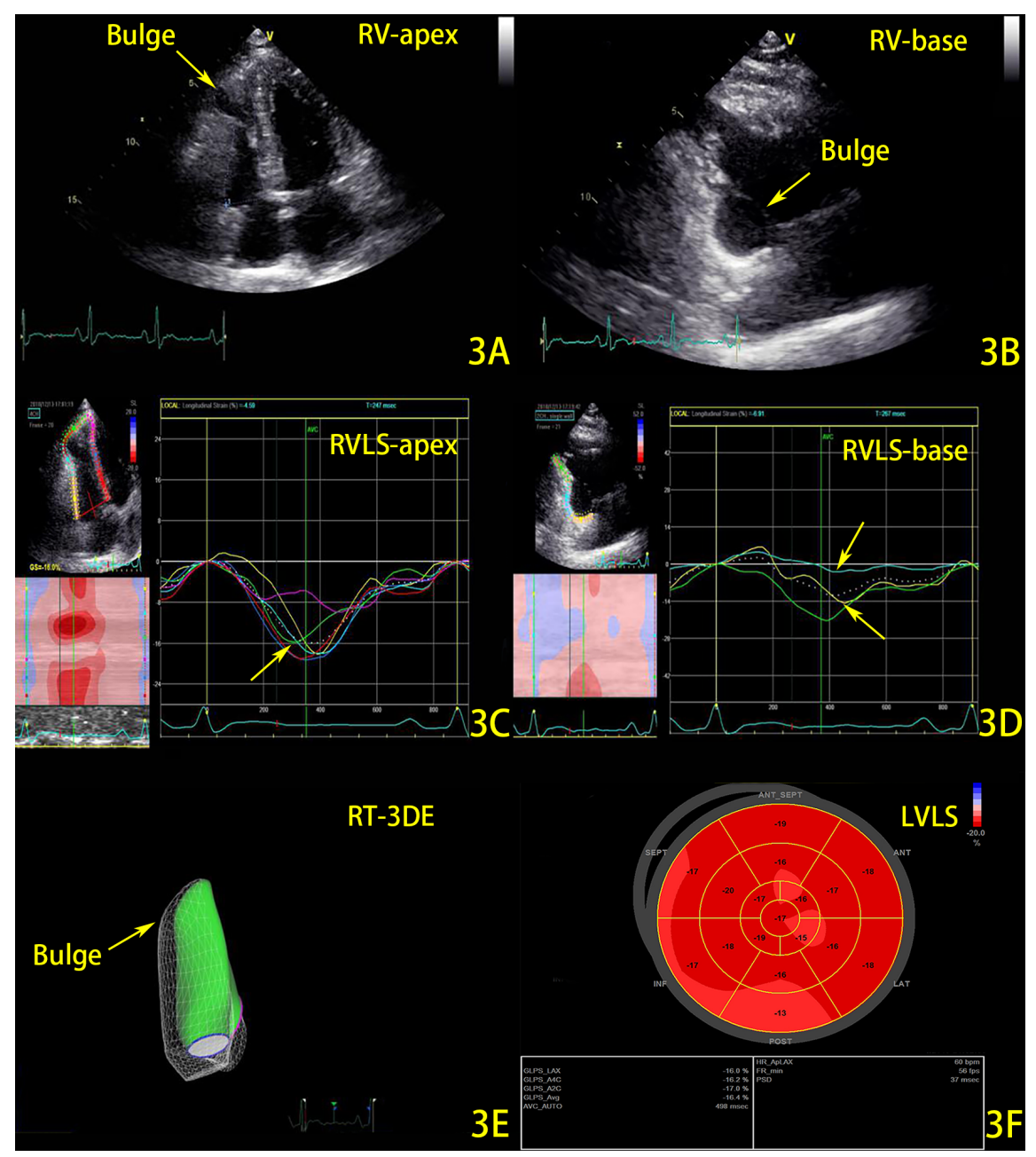




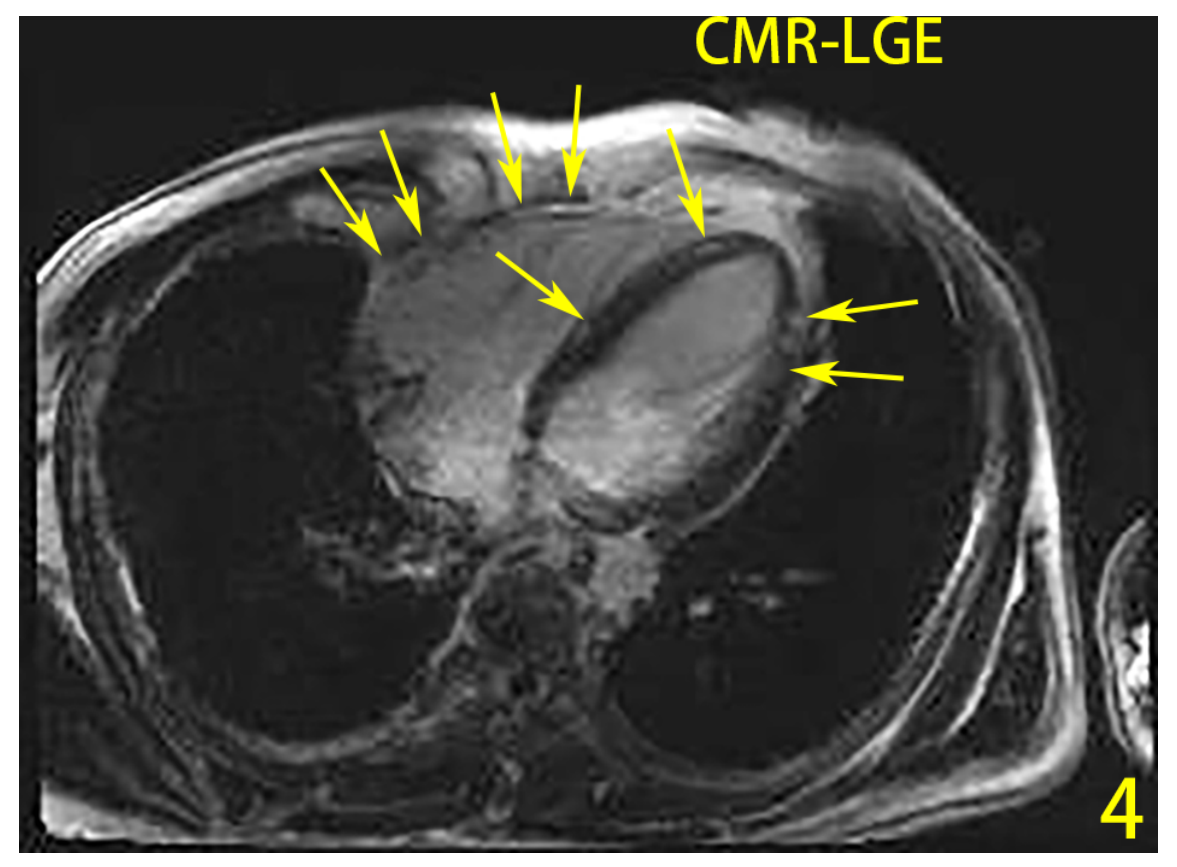

